\title{
reciamuc
}

Revista cientifica de investigación actualización del mundo de las ciencias

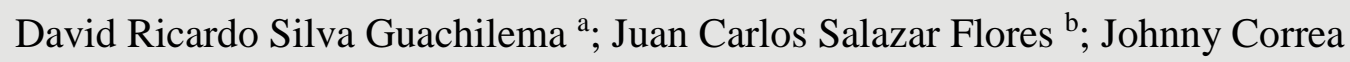
Michilena ${ }^{c}$; Javier Stalin Villagomez Toral

Dismenorrea y endometriosis. Como aliviar el dolor y cuando preocuparse

Dysmenorrhe and endometriosis. How to relieve pain and when to worry

Revista Científica de Investigación actualización del mundo de las Ciencias. Vol. 3 núm., 1, enero, ISSN: 2588-0748, 2018, pp. 78-94

DOI: $10.26820 / \mathrm{reciamuc/3.(1).enero.2019.78-94}$

URL: http://reciamuc.com/index.php/RECIAMUC/article/view/224

Código UNESCO: 3205 Medicina Interna

Tipo de Investigación: Artículo de Revisión

Editorial Saberes del Conocimiento

Recibido: 10/12/2018

Aceptado: 20/01/2019

Publicado: 30/01/2019

Correspondencia: daricsilvag@hotmail.com
a. Médico; daricsilvag@ hotmail.com
b. Médico; juank8sf@yahoo.es
c. Médico; johnnycorreamichilena@outlook.com
d. Médico; javime8590@outlook.com 


\section{Dismenorrea y endometriosis. Como aliviar el dolor y cuando preocuparse}

Vol. 3, núm. 1., (2019)

David Ricardo Silva Guachilema; Juan Carlos Salazar Flores; Johnny Correa Michilena; Javier Stalin Villagomez Toral

\section{RESUMEN}

La dismenorrea se define como los síntomas asociados con la menstruación, como dolor abdominal, calambres y lumbago, que interfieren con la actividad diaria. Dos causas comunes en el dolor pélvico en muchas mujeres son la dismenorrea primaria y la endometriosis. El mejor tratamiento de la dismenorrea primaria es la inhibición de la prostaglandina por los antiinflamatorios no esteroides (AINE) pero también se puede tratar con manipulación hormonal o cirugía. La endometriosis es una enfermedad ginecológica crónica caracterizada por síntomas dolorosos que son responsables de una disminución en la calidad de vida de los enfermos. El tratamiento de la endometriosis es, por lo tanto, un desafío para los ginecólogos y pacientes, ya que deben seleccionar el mejor enfoque terapéutico para esta enfermedad, sin embargo, se ha obtenido una mejor calidad de vida en estos pacientes con el uso del tratamiento convencional. La endometriosis es una enfermedad ginecológica crónica caracterizada por síntomas dolorosos que son responsables de una disminución en la calidad de vida de los enfermos.

Palabras Claves: Dismenorrea; Endometriosis; Dolor pélvico; Ginecología. 


\title{
Dismenorrea y endometriosis. Como aliviar el dolor y cuando preocuparse
}

Vol. 3, núm. 1., (2019)

David Ricardo Silva Guachilema; Juan Carlos Salazar Flores; Johnny Correa Michilena; Javier Stalin Villagomez Toral

\begin{abstract}
Dysmenorrhea is defined as the symptoms associated with menstruation, such as abdominal pain, cramps, and low back pain, which interfere with daily activity. Two common causes of pelvic pain in many women are primary dysmenorrhoea and endometriosis. The best treatment for primary dysmenorrhea is the inhibition of prostaglandin by nonsteroidal anti-inflammatory drugs (NSAIDs) but it can also be treated with hormonal manipulation or surgery. Endometriosis is a chronic gynecological disease characterized by painful symptoms that are responsible for a decrease in the quality of life of patients. The treatment of endometriosis is, therefore, a challenge for gynecologists and patients, since they must select the best therapeutic approach for this disease, however, a better quality of life has been obtained in these patients with the use of the treatment conventional. Endometriosis is a chronic gynecological disease characterized by painful symptoms that are responsible for a decrease in the quality of life of patients.
\end{abstract}

Key Words: Dysmenorrhea; Endometriosis; Pelvic pain; Gynecology. 


\section{Dismenorrea y endometriosis. Como aliviar el dolor y cuando preocuparse}

Vol. 3, núm. 1., (2019)

David Ricardo Silva Guachilema; Juan Carlos Salazar Flores; Johnny Correa Michilena; Javier

Stalin Villagomez Toral

\section{Introducción.}

La endometriosis es una enfermedad ginecológica caracterizada por La presencia de tejido endometrial fuera de la cavidad uterina. El tratamiento consiste en aliviar el dolor pélvico crónico (PPC por sus siglas en ingles) y recuperación de la fertilidad, a través de medicación y/o cirugía(Nácul, 2010).Los pacientes con endometriosis presentan los siguientes tipos del dolor: dolor pelvicocronico, dismenorrea, dispareunia, disquecia y disuria.

El dolor pélvico crónico CPP se define como dolor no menstrual o no cíclico, que dura al menos seis meses, lo suficientemente fuerte como para interferir con las actividades diarias y que requiere asistencia médica o tratamiento quirúrgico. La dismenorrea, también conocida como calambres menstruales es el dolor pélvico que se presenta antes o durante un período menstrual. El dolor durante las relaciones sexuales se llama dispareunia y el dolor cuando se defeca y orinan se conoce como disquecia y disuria, respectivamente. Como la enfermedad y el dolor son condiciones crónicas, existe una interferencia significativa en la calidad de vida de estas mujeres, en su desempeño profesional, y costos significativos para los servicios de salud. Respecto a la actividad profesional, un estudio multicéntrico mostró que los síntomas de la endometriosis tienen un impacto negativo en la productividad en el trabajo, con la pérdida de aproximadamente un día laborable por semana(Nnoaham \& Webster, 2011)

Otro estudio mostró que el $85 \%$ de los pacientes con endometriosis percibieron una disminución evidente en la calidad de su trabajo, el 19\% informó no poder trabajar debido al dolor y el $69 \%$ de los pacientes informaron que continúan trabajando a pesar de la sensación de dolor(Fourquet, y otros, 2010) 


\section{Dismenorrea y endometriosis. Como aliviar el dolor y cuando preocuparse}

Vol. 3, núm. 1., (2019)

David Ricardo Silva Guachilema; Juan Carlos Salazar Flores; Johnny Correa Michilena; Javier Stalin Villagomez Toral

Con respecto al gasto sanitario, existe la necesidad de cirugía para el diagnóstico definitivo de la enfermedad o incluso para evaluar las recurrencias, así como las hospitalizaciones de bidasal dolor. Los altos costos han sido asociados con gravedad de la endometriosis, la presencia de dolor pélvico, infertilidad, y una gran cantidad de años antes del diagnóstico.

Dado lo anterior, los síntomas de esta dolorosa condición ginecológica interfieren con la vida profesional y personal de los pacientes y, por tanto, el control de esta variable es esencial para proporcionar una mejor calidad de vida de esas mujeres. Como tal, este estudio tiene como objetivo evaluar los niveles de dolor en mujeres con endometriosis, centrándose en la influencia del tratamiento para controlar esta variable.

\section{Metodología.}

Para el desarrollo de este proceso investigativo, se plantea como metodología la encaminada hacia una orientación científica particular que se encuentra determinada por la necesidad de indagar en forma precisa y coherente una situación, en tal sentido (Davila, 2015) define la metodología "como aquellos pasos previos que son seleccionados por el investigador para lograr resultados favorables que le ayuden a plantear nuevas ideas".(p.66)

Lo citado por el autor, lleva a entender que el desarrollo de la acción investigativa busca simplemente coordinar acciones enmarcadas en una revisión bibliográfica con el fin de complementar ideas previas relacionadas a la dismenorrea y endometriosis a través de 


\section{Dismenorrea y endometriosis. Como aliviar el dolor y cuando preocuparse}

Vol. 3, núm. 1., (2019)

David Ricardo Silva Guachilema; Juan Carlos Salazar Flores; Johnny Correa Michilena; Javier Stalin Villagomez Toral

una revisión de literatura, para así finalmente elaborar un cuerpo de consideraciones generales que ayuden a ampliar el interés propuesto.

Tipo de Investigación

Dentro de toda práctica investigativa, se precisan acciones de carácter metodológico mediante las cuales, se logra conocer y proyectar los eventos posibles que la determinan, así como las características que hacen del acto científico un proceso interactivo ajustado a una realidad posible de ser interpretada. En este sentido, se puede decir, que la presente investigación corresponde al tipo documental, definido por Castro (2016), "se ocupa del estudio de problemas planteados a nivel teórico, la información requerida para abordarlos se encuentra básicamente en materiales impresos, audiovisuales y /o electrónicos”. (p.41).

En consideración a esta definición, la orientación metodológica permitió la oportunidad de cumplir con una serie de actividades inherentes a la revisión y lectura de diversos documentos donde se encontraron ideas explicitas relacionadas con los tópicos encargados de identificar a cada característica insertada en el estudio. Por lo tanto, se realizaron continuas interpretaciones con el claro propósito de revisar aquellas apreciaciones o investigaciones propuestas por diferentes investigadores relacionadas con el tema de interés, para luego dar la respectiva argumentación a los planteamientos, en función a las necesidades encontradas en la indagación.

\section{Fuentes Documentales}

El análisis correspondiente a las características que predomina en el tema seleccionado, llevan a incluir diferentes fuentes documentales encargadas de darle el respectivo apoyo y en ese 


\section{Dismenorrea y endometriosis. Como aliviar el dolor y cuando preocuparse}

Vol. 3, núm. 1., (2019)

David Ricardo Silva Guachilema; Juan Carlos Salazar Flores; Johnny Correa Michilena; Javier Stalin Villagomez Toral

sentido cumplir con la valoración de los hechos a fin de generar nuevos criterios que sirven de referencia a otros procesos investigativos. Para (CASTRO, 2016) las fuentes documentales incorporadas en la investigación documental o bibliográfica, "representa la suma de materiales sistemáticos que son revisados en forma rigurosa y profunda para llegar a un análisis del fenómeno".(p.41). Por lo tanto, se procedió a cumplir con la realización de una lectura previa determinada para encontrar aquellos aspectos estrechamente vinculados con el tema, con el fin de explicar mediante un desarrollo las respectivas apreciaciones generales de importancia.

\section{Técnicas para la Recolección de la Información}

La conducción de la investigación para ser realizada en función a las particularidades que determinan a los estudios documentales, tiene como fin el desarrollo de un conjunto de acciones encargadas de llevar a la selección de técnicas estrechamente vinculadas con las características del estudio. En tal sentido, (Bolívar, 2015), refiere, que es "una técnica particular para aportar ayuda a los procedimientos de selección de las ideas primarias y secundarias”. (p. 71).

Por ello, se procedió a la utilización del subrayado, resúmenes, fichaje, como parte básica para la revisión y selección de los documentos que presentan el contenido teórico. Es decir, que mediante la aplicación de estas técnicas se pudo llegar a recoger informaciones en cuanto a la revisión bibliográfica de los diversos elementos encargados de orientar el proceso de investigación. Tal como lo expresa, (Bolivar, 2015) "las técnicas documentales proporcionan las herramientas esenciales y determinantes para responder a los objetivos formulados y llegar a resultados efectivos" (p. 58). Es decir, para responder con eficiencia a las necesidades investigativas, se introdujeron como técnica de recolección el método inductivo, que hizo posible 


\section{Dismenorrea y endometriosis. Como aliviar el dolor y cuando preocuparse}

Vol. 3, núm. 1., (2019)

David Ricardo Silva Guachilema; Juan Carlos Salazar Flores; Johnny Correa Michilena; Javier Stalin Villagomez Toral

llevar a cabo una valoración de los hechos de forma particular para llegar a la explicación desde una visión general.

Asimismo, se emplearon las técnicas de análisis de información para la realización de la investigación que fue ejecutada bajo la dinámica de aplicar diversos elementos encargados de determinar el camino a recorrer por el estudio, según, (Bolívar, 2015) las técnicas de procesamiento de datos en los estudios documentales "son las encargadas de ofrecer al investigador la visión o pasos que debe cumplir durante su ejercicio, cada una de ellas debe estar en correspondencia con el nivel a emplear" (p. 123). Esto indica, que para llevar a cabo el procesamiento de los datos obtenidos una vez aplicado las técnicas seleccionadas, tales como: fichas de resumen, textual, registros descriptivos entre otros, los mismos se deben ajustar al nivel que ha sido seleccionado.

\section{Resultados.}

La dismenorrea se define como síntomas patológicos asociados con la menstruación, marcada por calambres abdominales y dolor durante el periodo menstrual que interfiere con la actividad diaria. Síntomas generales asociados, tales como náuseas, vómitos, lumbago, diarrea y dolor de cabeza también son comunes. La dismenorrea se clasifica en dos tipos, primarios y secundarios. La dismenorrea primaria se refiere al dolor menstrual sin patología subyacente, mientras que la dismenorrea secundaria se refiere a la menstruación dolorosa asociado a patología subyacente (tabla 1).

Un estudio, realizado con el apoyo del Ministerio de Salud y Bienestar Social de Japón, reveló que los dolores menstruales que requiere medicación para el dolor se produce en el 33\% 


\section{Dismenorrea y endometriosis. Como aliviar el dolor y cuando preocuparse}

Vol. 3, núm. 1., (2019)

David Ricardo Silva Guachilema; Juan Carlos Salazar Flores; Johnny Correa Michilena; Javier Stalin Villagomez Toral

de la menstruación de mujeres japonesas. En el 6\% de las mujeres, los analgésicos eran ineficaces y se necesitaba reposo en cama. Los resultados del estudio sugieren que 1/3 de las mujeres que menstrúan pueden requerir intervención médica(Taketani, Tsutsumi, Terakawa, \& Hoshiai, 2013).

La causa más frecuente de dismenorrea secundaria es la endometriosis. La endometriosis es una enfermedad inflamatoria estrogena dependiente caracterizada por el crecimiento ectópico de estroma y glándulas endometriales que afectan del 5\% al $15 \%$ de las mujeres en edad reproductiva. Sus características clínicas principales son dismenorrea, dolor pélvico crónico e infertilidad. La endometriosis es una enfermedad crónica y recurrente que adversamente afecta la calidad de vida en mujeres en edad reproductiva.

La teoría de la implantación de Sampson, que describe reflujo menstrual intraperitoneal que conduce al derrame de células endometriales en la cavidad peritoneal y el resultante desarrollo de lesiones endometriales, es actualmente la explicación predominante de la fisiopatología de la endometriosis. Mayor exposición a la menstruación en términos de ciclos menstruales cortos, flujo prolongado y baja paridad son posibles factores de riesgo(Eskenazi \& Warner, 1997) Por lo tanto, menstruación retrógrada observada en el $90 \%$ de las mujeres que menstrúan se considera un factor clave en la patogenia de la endometriosis.

Se cree que la incidencia de endometriosis está aumentando debido a los cambios en el estilo de vida de las mujeres, como la tendencia a casarse más tarde y tener menos hijos. Este cambio de estilo de vida aumenta el número de veces que una mujer menstrúa y afecta la incidencia de endometriosis. 
Dismenorrea y endometriosis. Como aliviar el dolor y cuando preocuparse

Vol. 3, núm. 1., (2019)

David Ricardo Silva Guachilema; Juan Carlos Salazar Flores; Johnny Correa Michilena; Javier Stalin Villagomez Toral

Tabla 1. Diagnóstico diferencial de dismenorrea primaria y secundaria

\begin{tabular}{lll}
\hline & Dismenorrea primaria & Dismenorrea secundaria \\
\hline Inicio & $\begin{array}{l}\text { Dentro de 3 años después } \\
\text { de la menarquia }\end{array}$ & $\begin{array}{l}\text { Mas de 5 años después de la } \\
\text { menarquia }\end{array}$ \\
Edad & $15-25$ años & Mas de 30 años \\
Envejecimiento & Mejora gradualmente & Empeora \\
Matrimonio & Mejora & No cambia \\
Posparto & Mejora & No cambia \\
\hline $\begin{array}{l}\text { Hallazgos de } \\
\text { endometriosis interna }\end{array}$ & la & $\begin{array}{l}\text { Endometriosis, Fibroma, } \\
\text { etc. }\end{array}$ \\
Tiempo & Menstruación & $\begin{array}{l}\text { Menstruación o un poco } \\
\text { mas si empeora }\end{array}$ \\
\hline Duración & $4-48$ horas & $1-5$ días \\
\hline
\end{tabular}

Mecanismo de dismenorrea primaria

La etiología de la dismenorrea primaria incluye un exceso o desequilibrio en la cantidad de secreción de prostaglandinas (PG) desde el endometrio durante la menstruación. Síntomas generales asociados, como náuseas, vómitos, lumbago, diarrea y cefalea son la secuela de la afluencia de PGs y sus derivados en circulación sistémica. La síntesis de los ácidos araquidónicos y la vía de la ciclooxigenasa se activan por una disminución de las concentraciones de progesterona en la fase secretora tardía. Los niveles de PG en el endometrio de la fase secretora tardía aumentan a niveles 3 veces más altos que los de la fase proliferativa. Las concentraciones de PGE2 y PGF2 alfa son mayores en el fluido menstrual de mujeres con 


\section{Dismenorrea y endometriosis. Como aliviar el dolor y cuando preocuparse}

Vol. 3, núm. 1., (2019)

David Ricardo Silva Guachilema; Juan Carlos Salazar Flores; Johnny Correa Michilena; Javier Stalin Villagomez Toral

dismenorrea que en mujeres con periodos sin dolor(Rees, 1989). Entre las PG, PGF2a es considerado el factor causal más potente del dolor.

Mecanismo del dolor debido a la endometriosis

Las concentraciones de PG son mayores en la sangre menstrual de mujeres con endometriosis. Se ha encontrado que la frecuencia, amplitud y el tono de la presión basal de las contracciones uterinas en mujeres con endometriosis fueron mayores que en los que no lo tienen. Así, la dismenorrea severa de pacientes con endometriosis puede ser el resultado de contracciones uterinas anormales.

Las lesiones y adherencias endometriales también pueden causar el dolor pélvico profundo asociado a la endometriosis. El dolor de los pacientes con endometriosis es por lo tanto debido tanto a la contracción uterina como a las lesiones endometrióticas.

\section{Endometriosis en mujeres jóvenes}

Estudios retrospectivos reportan un diagnóstico de endometriosisen $25 \%$ a $38 \%$ de los adolescentes con dolor pélvico crónico. Medicamentos antiinflamatorios no esteroides (AINE) y los anticonceptivos orales se prescriben para pacientes adolescentes con enfermedades de dolor pélvicocrónico. Dolor pélvico. Si estos agentes son refractarios, la endometriosisha sido diagnosticado en el 50\% al 70\% de estos pacientes (Laufer, 1997).

Estos resultados sugieren que la endometriosis debe ser parte del diagnóstico diferencial del dolor de pelvis crónica o abdominal inferior en niñas premenarqueales y perimenárquicas. 


\section{Dismenorrea y endometriosis. Como aliviar el dolor y cuando preocuparse}

Vol. 3, núm. 1., (2019)

David Ricardo Silva Guachilema; Juan Carlos Salazar Flores; Johnny Correa Michilena; Javier Stalin Villagomez Toral

Mientras que la endometriosis suele ocurrir en mujeres después de la menarquia, una serie de 5 niñas premenarqueales (entre 8,5 años y 13 años) se informó que tenían endometriosis(Marsh \& Laufer, 2005).

Niñas, que han tenido dolor pélvico crónico y un resultado negativo en el estudio gastrointestinal, se encontró que tenían lesiones endometriales peritoneales clasificadas por la American Society de la medicina reproductiva. Resección laparoscópica o la cauterización de todas las lesiones visibles resultó en una mejoría marcada de su dolor pélvico.

Aunque la teoría de Sampson sobre la menstruación retrógrada se cree que la implantación explica la mayoría de los casos de la endometriosis, esta teoría claramente no puede explicar los casos de endometriosis en niñas premenarqueales que ni menstrúan ni tienen reflujo retrógrado.

\section{Sintomas de la endometriosis en mujeres jóvenes}

Los síntomas comunes en mujeres con endometriosis incluyen dismenorrea, dolor abdominal inferior y dispareunia. Casi la mitad de las mujeres con endometriosis también reportan su incapacidad para quedar embarazada. El dolor de la endometriosis en adultos suele ser cíclica, mientras que la presentación del dolor pélvico de los adolescentes puede ser acíclico ocíclico. Los síntomas del intestino y la vejiga ocurren comúnmente enadolescentes (tabla 2). La Asociación de Endometriosis registro en 1998 de 4.000 mujeres adultas con endometriosis que 2/3 de los que respondieron a la encuesta experimentaron sus primeros síntomas pélvicos antes de los 20 años de edad, 21\% antes de los 15, y 17\% entre 15 y 19(Ballweg, 2003) 


\section{Dismenorrea y endometriosis. Como aliviar el dolor y cuando preocuparse}

Vol. 3, núm. 1., (2019)

David Ricardo Silva Guachilema; Juan Carlos Salazar Flores; Johnny Correa Michilena; Javier Stalin Villagomez Toral

\section{Tabla 2. Mecanismo de dolor asociado con endometriosis}

\section{Un dolor debido a lesiones endometriales.}

1. las lesiones peritoneales inducen reacciones inflamatorias y segregan.

Prostaglandinas, citoquinas, histamina y quinina que causan.

dolor.

2. la endometriosis infiltrante profunda destruye los tejidos y los nervios.

3. Los quistes de chocolate rotos pueden irritar el peritoneo

\section{Cicatriz y fibrosis, lesiones secundarias.}

1. Cicatriz, fibrosis, tracción y adhesión pueden reducir la movilidad de órganos El dolor puede ocurrir durante el movimiento u ovulación.

\section{La adherencia del intestino puede causar dolor de la defecación o disquecia.}

3. Útero retrovertido por adherencia, adherencia severa de ovarios.

a la bolsa de Douglas, y la induración del ligamento sacro puede causar disquecia.

\section{Diagnóstico de la endometriosis en mujeres jóvenes}

La inspección visual por laparoscopia o laparotomía es el estándar de oro para el diagnóstico de la endometriosis. Sin embargo, la laparoscopia no siempre se puede realizar en la práctica diaria. Por lo tanto, el término "endometriosis clínica” se utiliza cuando sólo la historia del paciente, el examen clínico y los ultrasonidos están disponibles para apoyar el diagnóstico. Los ginecólogos certificados pueden diagnosticar correctamente la endometriosis en el $80 \%$ de los pacientes sin laparoscopia. El diagnóstico diferencial es desafiante en adolescentes porque pueden existir enfermedades coexistentes, incluyendo los del sistema gastrointestinal y del tracto urinario, enfermedad inflamatoria pélvica, quistes ováricosobstructivos, anomalías y embarazo. Los síntomas de presentación pueden también variar, variando desde solo dismenorrea, dolor abdominal localizado, dispareunia, dolor no cíclico o combinaciones de estos síntomas. 


\section{Dismenorrea y endometriosis. Como aliviar el dolor y cuando preocuparse}

Vol. 3, núm. 1., (2019)

David Ricardo Silva Guachilema; Juan Carlos Salazar Flores; Johnny Correa Michilena; Javier Stalin Villagomez Toral

Durante la evaluación del paciente, el tumor de ovario y anomalías del tracto genital debe ser descartado. Se realiza un ultrasonido transabdominal mientras el paciente tiene una vejiga llena y descarta anomalías en el útero y ovarios. Luego se inspecciona el área genital y la vagina, y se realiza un examen rectal si es necesario. Las imágenes por resonancia magnética (MRI) también pueden ayudar a revelar tumor de ovario o cualquier otra anomalia.

\section{Resultados laparoscópicos en endometriosis en mujer joven}

Las lesiones endometrióticas consisten en una variedad de tipos, incluyendo implantes peritoneales, quistes ováricos de chocolate, adherencias, y endometriosis infiltrada profunda. Endometrioma ovárica es una lesión de enfermedad común que ocurre entre el 17\% a 44\% de los pacientes con endometriosis(Busacca \& Vignali, 2003). Sin embargo, el hallazgo es raro entre adolescentes y mujeres jóvenes.

Una revisión en un período de 15 años no informó endometriomasentre las masas ováricas encontradas en 102 lactantes, niños y adolescentes. Las lesiones de endometrismo peritoneal se clasifican en 3 subgrupos: rojo, negro y blanco.(Davis, 1993) Informó que las lesiones rojas son las predominantes en pacientes adolescentes. Examinadas 36 pacientes adolescentes que presentaron desmenorrea grave que fueron refractarias a la terapia previa, en comparación con 46 mujeres de 31 a 46 años, significativamente más lesiones rojas estaban presentes en los adolescentes.

Aunque no se ha encontrado correlación entre la severidad de los síntomas de dolor y la etapa de la enfermedad o sitio de las lesiones endometrióticas, la resección laparoscópica y el 


\section{Dismenorrea y endometriosis. Como aliviar el dolor y cuando preocuparse}

Vol. 3, núm. 1., (2019)

David Ricardo Silva Guachilema; Juan Carlos Salazar Flores; Johnny Correa Michilena; Javier Stalin Villagomez Toral

cauterio de las lesiones mejora los síntomas en más del 80\%de pacientes. Las lesiones claras son comunes en la endometriosis adolescente pero a menudo son difíciles de visualizar y evaluar.

\section{Gestión clínica de la endometriosis en mujeres jóvenes}

El tratamiento de la endometriosis puede ser quirúrgico o médico. El tratamiento quirúrgico, especialmente cirugía laparoscópica es el método más efectivo para controlar el dolor. La historia y el examen cuidadoso es necesario para descartar un tumor en la pelvis o enfermedad inflamatoria. Las mujeres jóvenes con dismenorrea deben tratarse inicialmente con una combinación de los AO y AINE cíclicos. Efectos secundarios, como náuseas y hemorragia atípica, suelen ocurrir durante los 3 primeros meses de terapia, y los pacientes deben ser informados sobre esta. Si la administración cíclica no es efectiva, el médico puede recomendar un régimen continuo de 3 a 4 meses en que el paciente toma la medicación, sin tener en cuenta cualquier sangrado de ruptura. Después de laparoscopia para los quistes ováricos, un régimen continuo de LEP es más efectivo para el dolor y resulta en menos lesiones recurrentes.

Después de la cirugía para los quistes ováricos de chocolate, si el dolor persiste con el manejo médico, la imagen incluyendo ultrasonido y resonancia magnética, se pueden ordenar para evaluar la endometriosis. La laparoscopia, es el estándar de oro para el diagnóstico, y la escisión laparoscópica de las lesiones son generalmente efectivas para pacientes con dolor severo.

La terapia agonista de la hormona liberadora de gonadotropina (GnRH) se usa ampliamente en pacientes adultos, pero no se recomienda como primera opción para las mujeres jóvenes porque la droga causa una reducción significativa en la densidad del mineral óseo. Una 


\section{Dismenorrea y endometriosis. Como aliviar el dolor y cuando preocuparse}

Vol. 3, núm. 1., (2019)

David Ricardo Silva Guachilema; Juan Carlos Salazar Flores; Johnny Correa Michilena; Javier Stalin Villagomez Toral

nueva progestina, Dienogest, ha estado disponible en Japón desde 2008 y puede ser utilizado para la gestión del dolor asociado a la endometriosis en mujeres jóvenes.

Dienogest tiene menos efectos secundarios debido al hipoestrógeno y se puede utilizar durante más de 1 año en pacientes adultos. Los datos no están disponibles para el agonista de GnRH y dienogest en una población adolescente. Estos agentes pueden ser introducidos después de establecer el diagnóstico de endometriosis por laparoscopia.

En resumen, la LEP y la cirugía laparoscópica son tratamientos de elección para pacientes jóvenes con endometriosis. La endometriosis es una enfermedad difícil de manejar debido a su naturaleza crónica y recurrente. Gestión a largo plazo, con especial atención a la preservación de la función ovárica, es muy importante en el tratamiento de mujeres jóvenes con endometriosis.

\section{Conclusiones.}

La evidencia apoya el uso de AINE tradicionales y terapia agonista de la hormona liberadora de gonadotropina $(\mathrm{GnRH})$ en el tratamiento de dolor asociado a dismenorrea primaria y endometriosis. Altos niveles de efectividad pueden ser esperados para ambos trastornos, lo que significa que muchas mujeres podrían encontrar alivio. Aunque el tratamiento tradicional para la endometriosis ha seguido un diagnóstico quirúrgico, también hay pruebas que apoyan el tratamiento empírico de la endometriosis en la mujer. Además de los AINE, existen varios medicamentos que pueden ayudar. Procedimientos invasivos y curas quirúrgicas deben reservarse para aquellos que no son mejorados por las terapias previamente descritas. 


\section{Dismenorrea y endometriosis. Como aliviar el dolor y cuando preocuparse}

Vol. 3, núm. 1., (2019)

David Ricardo Silva Guachilema; Juan Carlos Salazar Flores; Johnny Correa Michilena; Javier

Stalin Villagomez Toral

\section{Bibliografía.}

Ballweg, M. (2003). Big picture of endometriosis helps provide guidance on approach to teens: comparative historical data show endo starting younger, is more severe. Pediatr Adolesc Gynecol, 21-26.

Bolívar, J. (2015). Investigación Documental. México. Pax.

Busacca, M., \& Vignali, M. (2003). Ovarian endometriosis: from pathogenesis. Curr Opin Obstet Gynecol. 2003(15), 321-326.

Castro, J. (2016). Técnicas Documentales. México. Limusa.

Davila, A. (2015). Diccionario de terminos cientificos. Caracas: Oasis.

Davis, G. (1993). Clinical characteristics of adolescent endometriosis. Adolescent Health, 362368.

Eskenazi, B., \& Warner, M. (1997). Epidemiology of endometriosis. Obstet Gynecol Clin North Am., 24-58.

Fourquet, J., Gao, X., Zavala, D., Orengo, J., Abac, S., \& Ruiz, A. (2010). Patients' report on how endometriosis affects health, work, and daily life. Fertil Steril.

Laufer, M. (1997). Prevalence of endometriosis in adolescent women with chronic pelvic pain not responding to conventional therapy. Pediatr Adolesc Gynecol, 199-202.

Marsh, E., \& Laufer, M. (2005). Endometriosis in premenarcheal girls who do not have an associated obstructive anomaly. Fertile Steril 2005(83), 758-760.

Nácul, A. S. (2010). Aspectos de diagnóstico y tratamiento de. Rev Bras Ginecol Obstet, 298307.

Nnoaham, K. H., \& Webster, P. (2011). Impact of endometriosis on quality of life and. Fertil Steril, 366-373.

Rees, M. (1989). Heavy painful periods. Balliere's Clin Obstet Gynacol.

Taketani, Y., Tsutsumi, O., Terakawa, N., \& Hoshiai, H. (30 de Junio de 2013). [2000 General report for the prevention, diagnosis and treatment of endometriosis in the aspect of reproductive health. Obtenido de http://mhlw-grants.niph.go.jp. 